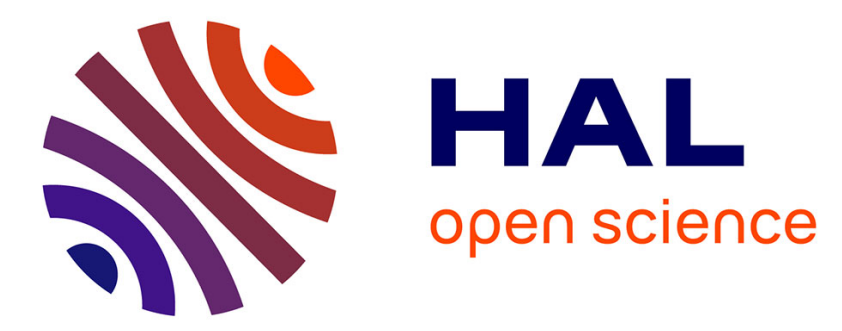

\title{
A class of two-fluid two-phase flow models
}

Frédéric Coquel, Jean-Marc Hérard, Khaled Saleh, Nicolas Seguin

\section{To cite this version:}

Frédéric Coquel, Jean-Marc Hérard, Khaled Saleh, Nicolas Seguin. A class of two-fluid two-phase flow models. 42nd AIAA Fluid Dynamics Conference and Exhibit, Jun 2012, New Orleans, United States. 10.2514/6.2012-3356 . hal-01582645

\section{HAL Id: hal-01582645 \\ https://hal.science/hal-01582645}

Submitted on 5 Apr 2018

HAL is a multi-disciplinary open access archive for the deposit and dissemination of scientific research documents, whether they are published or not. The documents may come from teaching and research institutions in France or abroad, or from public or private research centers.
L'archive ouverte pluridisciplinaire HAL, est destinée au dépôt et à la diffusion de documents scientifiques de niveau recherche, publiés ou non, émanant des établissements d'enseignement et de recherche français ou étrangers, des laboratoires publics ou privés. 


\title{
A Class of Two-Fluid Two-Phase Flow Models
}

\author{
Frédéric Coquel* \\ CMAP, Ecole Polytechnique, Route de Saclay, 91128, Palaiseau, France. \\ Jean-Marc Hérard ${ }^{\dagger}$ \\ EDF, RED, 6 quai Watier, 78400, Chatou, France. \\ Khaled Saleh $\ddagger$ \\ EDF, RED, 6 quai Watier, 78400, Chatou, France. \\ Nicolas Seguin ${ }^{\S}$ \\ LJLL, Université Pierre et Marie Curie, 4 place Jussieu, 75005, Paris, France.
}

\begin{abstract}
We introduce a class of two-fluid models that complies with a few theoretical requirements that include : (i) hyperbolicity of the convective subset, (ii) entropy inequality, (iii) uniqueness of jump conditions for non-viscous flows. These specifications are necessary in order to compute relevant approximations of unsteady flow patterns. It is shown that the Baer-Nunziato model belongs to this class of two-phase flow models, and the main properties of the model are given, before showing a few numerical experiments.
\end{abstract}

\section{Introduction}

The Baer-Nunziato model (called BN model afterwards) was introduced in the early eighties in order to provide a suitable representation of gas-particle granular flows, when compressible effects cannot be neglected, and more precisely in order to tackle deflagration to detonation transition. This model has been examined in detail since the early paper ${ }^{4}$; we must at least mention Kapila and co-workers $\left({ }^{5,22,23}\right)$, Gavrilyuk and Saurel, ${ }^{12}$ but also Glimm and co-workers, ${ }^{14,15,21}$ among others ( $\mathrm{see}^{24,25,28}$ also). Many papers have been devoted to the numerical simulation of this model, among which we may point out those by Saurel and Abgrall, ${ }^{30}$ Gonthier and Powers, ${ }^{16}$ Toro, ${ }^{33}$ Andrianov and Warnecke, ${ }^{3}$ Lowe, ${ }^{27}$ Schwendemann et al, ${ }^{31}$ who proposed various approximate Riemann solvers, but also Coquel et al, ${ }^{1,2}$ who suggested to use relaxation schemes as a keystone for such a purpose. Some recent computational results can be found in $^{32}$ and ${ }^{19}$ for instance.

It was in fact shown in ${ }^{6,11}$ that the BN model is one among a few two-fluid models that benefit from several essential properties. Actually, noting as usual $\alpha_{k}$ (such that $\alpha_{l}+\alpha_{v}=1$ ), $U_{k}, \rho_{k}, m_{k}=\alpha_{k} \rho_{k}, E_{k}$ and $P_{k}$ the statistical void fractions, velocities, densities, partial masses, total energies and pressures respectively (for $k=l, v$, where $l$ and $v$ subscripts respectively refer to the liquid and vapour phases), but also:

$$
W=\left(\alpha_{l}, m_{l}, m_{v}, m_{l} U_{l}, m_{v} U_{v}, \alpha_{l} E_{l}, \alpha_{v} E_{v}\right)
$$

\footnotetext{
${ }^{*}$ Research Director in CNRS, CMAP, UMR 7671, Ecole Polytechnique, Route de Saclay, 91128, Palaiseau, France.

†Senior engineer, EDF R\&D, Fluid Dynamics, Power Generation and Environment, 6 quai Watier, 78400, Chatou, France.

${ }^{\ddagger}$ PhD student, EDF, R\&D, Fluid Dynamics, Power Generation and Environment, 6 quai Watier, 78400, Chatou, France, and: Laboratoire Jacques-Louis Lions, UMR 7598, 4 place Jussieu, 75005, Paris, France.

$\S$ Associate Professor, Laboratoire Jacques-Louis Lions, Université Pierre et Marie Curie, UMR 7598, 4 place Jussieu, 75005 , Paris, France.
} 
and starting from the open set of PDEs, for $k=l, v$ :

$$
\left\{\begin{array}{l}
\partial_{t}\left(\alpha_{k}\right)+V_{i}(W) \partial_{x}\left(\alpha_{k}\right)=\phi_{k}(W) \\
\partial_{t}\left(\alpha_{k} \rho_{k}\right)+\partial_{x}\left(\alpha_{k} \rho_{k} U_{k}\right)=0 ; \\
\partial_{t}\left(\alpha_{k} \rho_{k} U_{k}\right)+\partial_{x}\left(\alpha_{k} \rho_{k} U_{k}^{2}\right)+\alpha_{k} \partial_{x}\left(P_{k}\right)+\left(P_{k}-P_{i}(W)\right) \partial_{x}\left(\alpha_{k}\right)=D_{k}(W) ; \\
\partial_{t}\left(\alpha_{k} E_{k}\right)+\partial_{x}\left(\alpha_{k} U_{k}\left(E_{k}+P_{k}\right)\right)+P_{i}(W) \partial_{t}\left(\alpha_{k}\right)=\psi_{k}(W)+V_{I} D_{k}(W) .
\end{array}\right.
$$

where $D_{k}(W)$ and $\psi_{k}(W)$ enable to take drag effects and heat transfer into account, authors of the latter reference introduced three distinct couples $\left(P_{i}(W), V_{i}(W)\right)$ which enable to achieve the following requirements:

- The two-fluid model is hyperbolic without any restriction on the space of physical states (other than those already existing for single-phase flow models);

- Smooth solutions of the whole set of partial differential equations are governed by a meaningful entropy inequality;

- Unique jump conditions can be exhibited within each isolated field;

- The model generates smooth solutions that comply with positivity constraints.

The first point is physically relevant, and it is indeed mandatory to compute solutions of a well-posed initial value problem, when tracking unsteady flow patterns. The second point is not only desirable from a physical point of view, but it also introduces a nice tool in order to control smooth but also shock solutions. The third one introduces an important difference with other classical two-fluid models, for instance those that assume a local instantaneous pressure equilibrium between phases: actually, this third property will also guarantee that (stable enough) schemes will converge towards the same solution when refining the mesh, which is of course implicitly assumed by users... We emphasize that these specifications have also been used in order to model granular flows and flows in porous media (see ${ }^{10,13,17}$ ). The BN model is suitable for many water-vapour flows, for instance for standard computations in the primary circuit of nuclear power reactors, since the liquid flow is expected to contain a very small amount of vapour bubbles in standard conditions. It is also relevant for some applications where the vapour phase is dominant and when few liquid droplets are present in the field. In the first case, the closure laws for the couple $\left(P_{i}(W), V_{i}(W)\right)$ should be $\left(P_{l}, U_{v}\right)$, and in the second case one should use $\left(P_{v}, U_{l}\right)$ reversely.

However, there are some applications where the BN model can hardly be used. This may happen in at least two distinct configurations:

- when the flow contains different regions in terms of topology at the beginning of the computation: this may happen in many practical cases;

- when some change occurs in the flow during the time interval which is of interest: this is the case for instance when heating a liquid flow through a wall boundary (this will correspond to the so-called boiling crisis in the nuclear safety framework).

These situations have led to the present proposition, which aims at providing a general framework which:

- complies with the four above-mentionned criteria;

- contains the BN model.

We present in the sequel this general framework. ${ }^{18}$ Next we detail the main properties of the two-fluid model. We eventually discuss a few numerical experiments that illustrate the whole approach. 


\section{Governing set of equations of the two-fluid model}

The new framework that is proposed in this paper introduces a non-dimensional scalar variable $\beta$ that characterizes the flow regime. This variable is lying in the interval [0,1]. Setting $m_{k}=\alpha_{k} \rho_{k}$, the governing set of equations reads:

$$
\left\{\begin{array}{l}
\partial_{t}(\beta)+\mathcal{W}(W, \beta) \partial_{x}(\beta)=T_{\beta}(W, \beta) \\
\partial_{t}\left(\alpha_{k}\right)+V_{i}(W, \beta) \partial_{x}\left(\alpha_{k}\right)=\phi_{k}(W) \\
\partial_{t}\left(m_{k}\right)+\partial_{x}\left(m_{k} U_{k}\right)=0 \\
\partial_{t}\left(m_{k} U_{k}\right)+\partial_{x}\left(m_{k} U_{k}^{2}\right)+\alpha_{k} \partial_{x}\left(P_{k}\right)+\left(P_{k}-P_{i}(W, \beta)\right) \partial_{x}\left(\alpha_{k}\right)=D_{k}(W) \\
\partial_{t}\left(\alpha_{k} E_{k}\right)+\partial_{x}\left(\alpha_{k} U_{k}\left(E_{k}+P_{k}\right)\right)+P_{i}(W, \beta) \partial_{t}\left(\alpha_{k}\right)=\psi_{k}(W)+\bar{V}_{I}(W) D_{k}(W)
\end{array}\right.
$$

for $k=l, v$, noting $E_{k}=\rho_{k}\left(e_{k}\left(P_{k}, \rho_{k}\right)+U_{k}^{2} / 2\right)$ the total energy within phase $k$, and assuming some relevant equation of state for $e_{k}\left(P_{k}, \rho_{k}\right)$. Terms on the right-hand side must follow the standard rule:

$$
\sum_{k=l, v} \psi_{k}(W)=0 \quad ; \quad \sum_{k=l, v} D_{k}(W)=0 \quad ; \quad \sum_{k=l, v} \phi_{k}(W)=0 .
$$

which means that these contributions account for interfacial transfer terms. The derivation of the governing open equation for $\alpha_{k}$ can be found in ${ }^{18}$; the reader is also refered to ${ }^{20}$ and $^{12}$ for that particular topic. Source terms $\phi_{k}(W), D_{k}(W), \psi_{k}(W)$ will be detailed in the next section, and we note here:

$$
\bar{V}_{I}(W)=\left(U_{l}+U_{v}\right) / 2 .
$$

The so-called interface velocity $V_{i}(W, \beta)$ will be defined according to:

$$
V_{i}(W, \beta)=\mu(W, \beta) U_{l}+(1-\mu(W, \beta)) U_{v}, \quad \text { with } \mu \in[0,1] .
$$

A straightforward consequence is that $U_{1}(x, t)=U_{2}(x, t)=U$ implies $V_{i}(x, t)=U$ locally. We will also assume that the following holds:

$$
T_{\beta}(W, \beta=0)=T_{\beta}(W, \beta=1)=T_{\beta}(W, \beta=1 / 2)=0,
$$

whatever $W$ would be.

\section{A. Closure laws for $P_{i}$ and interfacial transfer terms}

If we note $c_{k}$ and $S_{k}$ the sound velocity and the specific entropy within phase $k$, we may introduce the entropy-entropy flux couple $\left(S, f_{S}\right)$ as follows :

$$
S=m_{l} S_{l}+m_{v} S_{v} ; \quad f_{S}=m_{l} S_{l} U_{l}+m_{v} S_{v} U_{v} .
$$

We also introduce temperatures $T_{k}$ such that :

$$
1 / T_{k}=\left(\partial_{P_{k}}\left(S_{k}\left(P_{k}, \rho_{k}\right)\right)\right)\left(\partial_{P_{k}}\left(e_{k}\left(P_{k}, \rho_{k}\right)\right)\right)^{-1},
$$

for $k=l, v$.

Using these notations, we will assume that closure laws for $\phi_{l}, \psi_{l}, D_{l}$ comply with the conditions:

$$
\left\{\begin{array}{l}
0 \leq \psi_{l}\left(T_{v}-T_{l}\right) \\
0 \leq \phi_{l}\left(P_{l}-P_{v}\right) \\
0 \leq D_{l}\left(U_{v}-U_{l}\right)
\end{array}\right.
$$

We keep closure laws for $\psi_{l}$ and $D_{l}$ that are in agreement with those given in the standard literature, ${ }^{20}$ setting:

$$
\left\{\begin{array}{l}
\psi_{l}=\frac{m_{l} m_{v}\left(C_{v}\right)_{v}\left(C_{v}\right)_{l}}{m_{l}\left(C_{v}\right)_{l}+m_{v}\left(C_{v}\right)_{v}}\left(T_{v}-T_{l}\right) / \tau_{T} \\
D_{l}=\frac{m_{l} m_{v}}{m_{l}+m_{v}}\left(U_{v}-U_{l}\right) / \tau_{U} .
\end{array}\right.
$$


where $\tau_{U}, \tau_{T}$ respectively denote velocity and temperature relaxation time scales. The closure law for $\phi_{l}$ is assumed to be non zero when $P_{v} \neq P_{l}$. A possible choice is:

$$
\phi_{l}=\alpha_{l} \alpha_{v}\left(P_{l}-P_{v}\right) / \Pi_{0} / \tau_{P}
$$

where $\tau_{P}$ represents the pressure relaxation time scale (see ${ }^{4,12}$ for instance), and $\Pi_{0}$ is a pressure reference.

Turning then to the interfacial pressure $P_{i}(W, \beta)$, we introduce:

$$
P_{i}(W, \beta)=\left((1-\mu(W, \beta)) P_{l} / T_{l}+\mu(W, \beta) P_{v} / T_{v}\right) /\left((1-\mu(W, \beta)) / T_{l}+\mu(W, \beta) / T_{v}\right)
$$

Actually, this closure law is mandatory in order to obtain a physically relevant entropy inequality. Hence the interface pressure is totally determined as soon as the interface velocity is prescribed. We recall that the same procedure applies when modelling three-phase flows $\left(\operatorname{see}^{17}\right)$. Obviously, the local balance $P_{v}=P_{l}=P$ will imply $P_{i}=P$.

\section{B. Closure laws for $V_{i}$ and $\mathcal{W}$}

We define : $\mathcal{W}(W, \beta)=\mathcal{W}_{0}$ or alternatively, $\mathcal{W}(W, \beta)=\mathcal{W}_{1}$ where:

$$
\mathcal{W}_{0}=0 \quad \text { and: } \quad \mathcal{W}_{1}=\left(m_{l} U_{l}+m_{v} U_{v}\right) /\left(m_{l}+m_{v}\right)
$$

and we introduce the interfacial velocity $V_{i}$ such that $\mu$ in (4) reads:

$$
\mu(\beta, W)=\frac{m_{l} \beta}{m_{l} \beta+m_{v}(1-\beta)} .
$$

We note that the specific value $\beta=0$ (respectively $\beta=1$ ) corresponds to the BN model, since the associated values of the interface pressure and interface velocity become $P_{i}=P_{l}$ and $V_{i}=U_{v}$ (respectively $P_{i}=P_{v}$ and $V_{i}=U_{l}$ ), owing to (9). The BN model is appealing for many scientists, since it guarantees that the interface velocity corresponds to the velocity of the vanishing phase, and meanwhile it complies with the expected idea that the interface presssure should be driven by the most present phase. Moreover, the value $\beta=1 / 2$ was already pointed out in; ${ }^{11}$ in that very special case $V_{i}$ and $\mathcal{W}_{1}$ identify. Obviously, when considering an initial condition such that $\beta(x, t=0)=0$ (respectively $\beta(x, t=0)=1$ ), an obvious solution of the first equation in (2) is simply : $\beta(x, t)=0$ (respectively $\beta(x, t)=1$ ). A similar remark holds for $\beta=1 / 2$. Within our nuclear framework, a typical situation where the initial condition may involve two seperate regions $\Omega_{A}$ and $\Omega_{B}$ with distinct values of $\beta$, typically $\beta\left(x \in \Omega_{A}, 0\right)=0$ on the one side and $\beta\left(x \in \Omega_{B}, 0\right)=1$ on the other side, is the LOCA situation (Loss Of Coolant Accident). The governing set of equations is closed now, assuming that relaxation time scales $\tau_{U}, \tau_{P}, \tau_{T}$ and $T_{\beta}(W, \beta)$ are given.

\section{Main properties of the two-fluid model}

We provide now the main properties of the two-fluid model:

Proposition 1:

Smooth solutions of (2) comply with the following entropy inequality:

$$
\partial_{t}(S)+\partial_{x}\left(f_{S}\right)=\Sigma_{k}\left(\psi_{k}+\left(\bar{V}_{I}-U_{k}\right) D_{k}-\phi_{k}\left(P_{i}-P_{k}\right)\right) / T_{k} \geq 0 .
$$

The proof is straightforward (see ${ }^{18}$ ). This entropy inequality enables to select physically relevant shocks in the non-viscous case. We may now give the following main result:

\section{Proposition 2:}

- System (2) is hyperbolic since it admits real eigenvalues:

$$
\lambda_{1}=V_{i}, \lambda_{2}=\mathcal{W}, \lambda_{3}=U_{l}, \lambda_{4}=U_{l}-c_{l}, \lambda_{5}=U_{l}+c_{l}, \lambda_{6}=U_{v}, \lambda_{7}=U_{v}-c_{v}, \lambda_{8}=U_{v}+c_{v} .
$$


and associated right eigenvectors span the whole space $\mathcal{R}^{8}$ if and only if:

$$
\left|V_{i}-U_{k}\right| \neq c_{k} \quad \text { and: } \quad\left|\mathcal{W}-U_{k}\right| \neq c_{k}
$$

for $k=l, v$. Otherwise, the resonance phenomenon occurs in the solution.

- Waves associated with $\lambda_{1}, \lambda_{2}, \lambda_{3}, \lambda_{6}$ are linearly degenerate and those corresponding to $\lambda_{4}, \lambda_{5}, \lambda_{7}, \lambda_{8}$ are genuinely nonlinear.

The most difficult part in the proof corresponds to the first claim in the second item $\left(\mathrm{see}^{18}\right)$.

\section{Proposition 3:}

Field by field jump conditions are uniquely defined in system (2), unless resonance occurs (if a GNL field overlaps with a LD field).

We note that for nuclear applications in pressurised water reactors, the resonance phenomenon is very unlikely to appear. However, even in that framework, we emphasize that shock waves may occur, due for instance to sudden high heating fluxes through wall boundaries, or due to modifications of inlet/outlet boundary conditions. Thus the third requirement is again relevant for these applications. Jump conditions actually coincide with single-phase jump conditions within each phase, on each side of the void fraction coupling wave associated with $\lambda=V_{i}$.

When focusing on solutions of the one-dimensional Riemann problem associated with the homogeneous part of (2), it appears that the contact discontinuity associated with $V_{i}$ separates both regions $\Omega_{L}=\left\{(x, t) / x / t<V_{i}\right\}$ where $\alpha_{l}(x, t)=\left(\alpha_{l}\right)_{L}$, and $\Omega_{R}=\left\{(x, t) / x / t>V_{i}\right\}$ where $\alpha_{l}(x, t)=\left(\alpha_{l}\right)_{R}$. In each subdomain $\Omega_{L, R}$, the jump relations are:

$$
\left\{\begin{array}{l}
-\sigma\left[\rho_{k}\right]_{a}^{b}+\left[\rho_{k} U_{k}\right]_{a}^{b}=0 \\
-\sigma\left[\rho_{k} U_{k}\right]_{a}^{b}+\left[\rho_{k} U_{k}^{2}+P_{k}\right]_{a}^{b}=0 \\
-\sigma\left[E_{k}\right]_{a}^{b}+\left[U_{k}\left(E_{k}+P_{k}\right)\right]_{a}^{b}=0
\end{array}\right.
$$

if $\sigma$ denotes the speed of the travelling shock wave separating states $a$ and $b$, for $k=l, v$. Note also that the solution $\beta(x, t)$ in the Riemann problem is given by $\beta(x, t)=(\beta)_{L}$ in the subdomain $\omega_{L}=\{(x, t) / x / t<\mathcal{W}\}$, and : $\beta(x, t)=(\beta)_{R}$ in the subdomain $\omega_{R}=\{(x, t) / x / t>\mathcal{W}\}$. Eventually, noting $\mathcal{D}$ the whole physical domain, we get the next expected result:

\section{Proposition 4:}

Assuming positive inlet boundary conditions and initial conditions for $\alpha_{l, v}$ and $m_{l, v}$, then smooth solutions of system (2) are such that void fractions $\alpha_{l, v}$ and partial masses $m_{l, v}$ remain positive over $\mathcal{D} \times[0, T]$.

\section{Numerical experiments}

We provide here a numerical experiment that illustrates the behaviour of the two-fluid model. Numerical schemes are those that are used in. ${ }^{19}$ We focus here on the particular choice $\mathcal{W}(W, \beta)=\mathcal{W}_{0}$, and $T_{\beta}(W, \beta)=-\beta\left(\beta^{2}-3 \beta / 2+1 / 2\right) / \tau_{\beta}(W)$ and we consider a very difficult test case, that is very unlikely to happen in our framework, since it includes the resonance phenomenon.

We consider a $1 \mathrm{D}$ computational domain $\mathcal{D}=[0,1]$, and set the initial discontinuity at the interface $x_{0}=0.5$. The initial values of the function $\beta$ are: $\beta\left(x<x_{0}, t=0\right)=0$, and $\beta\left(x>x_{0}, t=0\right)=1$. Thus it means that we assume that the flow on the left side (or left code) $x<0.5$ is modeled with the BN model corresponding to $\left(P_{i}, U_{i}\right)=\left(P_{l}, U_{v}\right)$, and that we have retained the couple $\left(P_{i}, U_{i}\right)=\left(P_{v}, U_{l}\right)$ on its right-hand side. The initial conditions are the following: 


\begin{tabular}{|c|c|c|}
\hline & Initial left state L & Initial right state R \\
\hline$\beta$ & 0 & 1 \\
\hline$\alpha_{l}$ & 0.98 & 0.02 \\
\hline$\alpha_{v}$ & 0.02 & 0.98 \\
\hline$\rho_{l}$ & 1 & 0.125 \\
\hline$\rho_{v}$ & 4 & 0.5 \\
\hline$U_{l}$ & \multicolumn{2}{|c|}{0.} \\
\hline$U_{v}$ & \multicolumn{2}{|c|}{0.} \\
\hline$P_{l}$ & $10^{5}$ & $10^{4}$ \\
\hline$P_{v}$ & $4 \times 10^{5}$ & $4 \times 10^{4}$ \\
\hline
\end{tabular}

Initial condition in test case.

Perfect gas EOS have been considered within each phase: $P_{l, v}=\left(\gamma_{l, v}-1\right) \rho_{l, v} e_{l, v}$, with $\gamma_{v}=1.2$ and $\gamma_{l}=1.2$. The flow is at rest at the beginning of the computation and time scales $\tau_{U}, \tau_{P}, \tau_{T}$ have been set to 1 , thus the solution is very close to the solution of a Riemann problem corresponding to : $\tau_{U}=\tau_{P}=\tau_{T}=+\infty$, since the final time of the computation is $T=10^{-3}$. We use two regular meshes with $10^{4}$ and $4 \times 10^{4}$ cells respectively. Two shock waves (one within each phase) are created and move to the right side of the interface. The liquid (resp. vapour) rarefaction wave is subsonic (resp. supersonic, see figure 1).
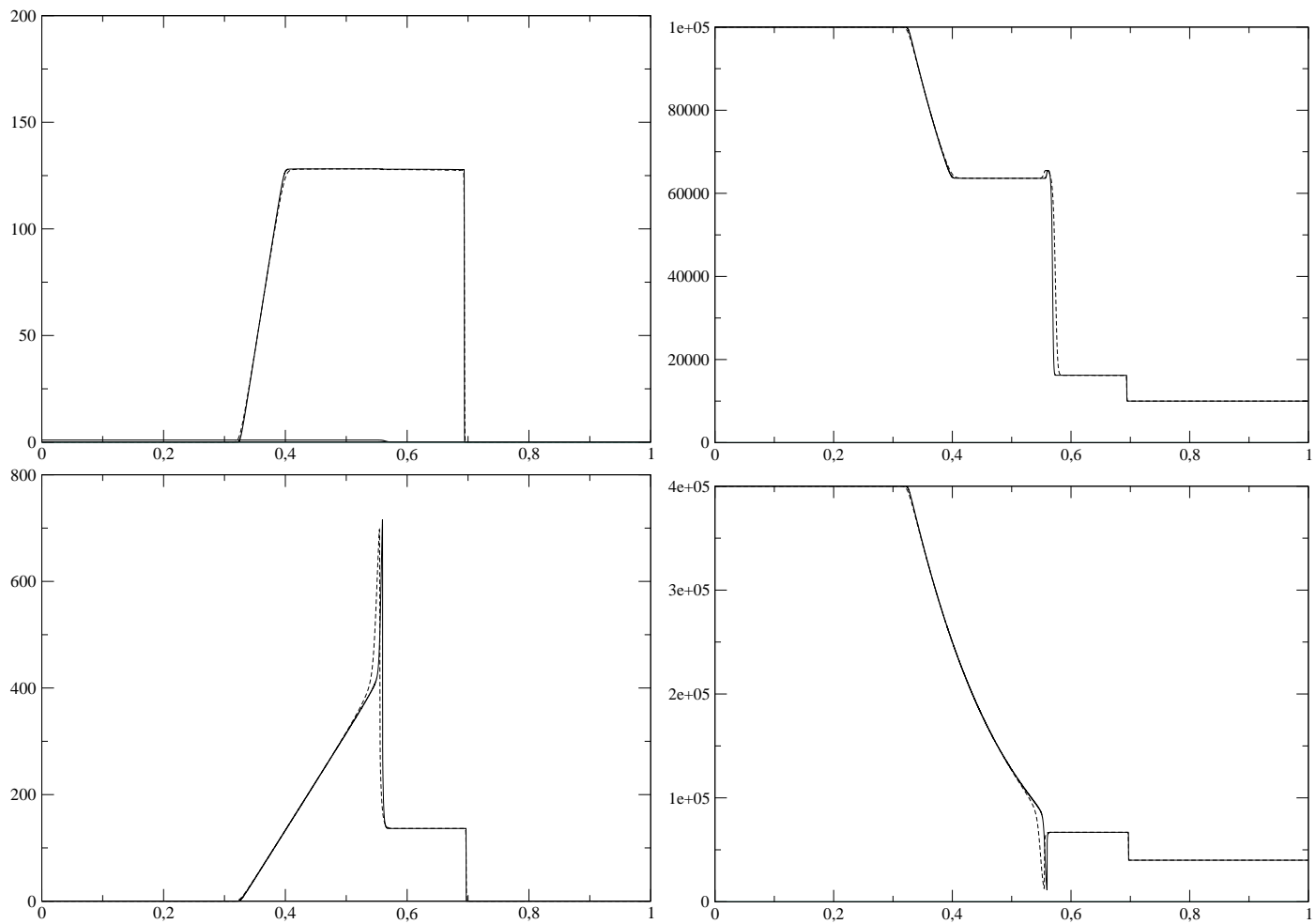

Figure 1. Velocity (left) and pressure (right) profiles for the vapour phase (down) and liquid phase (top) respectively. The two regular meshes contain 10000 (dashed line) and 40000 (plain line) cells.

\section{Conclusion}

The general class of two-fluid models that has been introduced herein may in fact be viewed as a symetrized dynamical version of the BN model. It contains a scalar function $\beta$ which specifies the flow regime. The main properties of the two-fluid model have been given, and more details can be found in the 


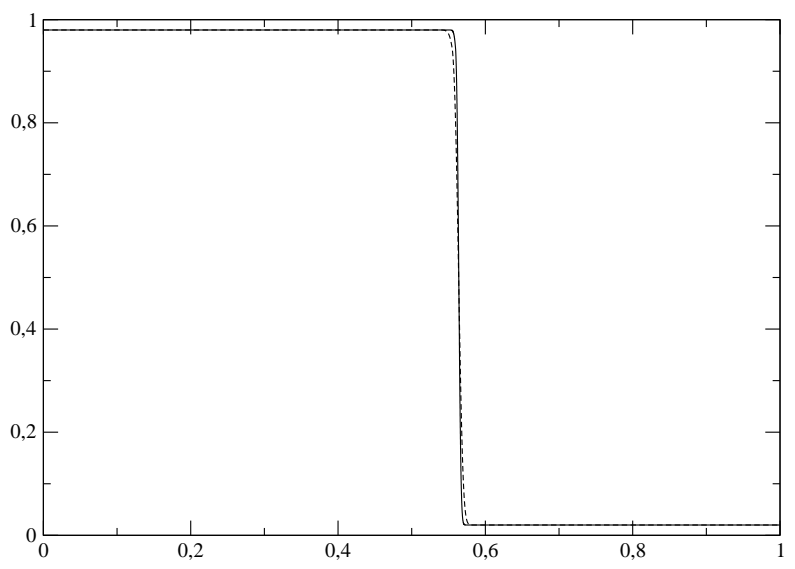

Figure 2. Void fraction profiles.

reference, ${ }^{18}$ some first numerical experiments have been achieved, but there is now of course a need for an extensive investigation and validation that requires a great amount of work. Among others, the fractional step method introduced in ${ }^{7}$ may be used for computational purposes, and we refer to this reference which gives numerical rates of convergence obtained while focusing on some particular Riemann problems. This method takes advantage of the LD structure of the 1 and 2-waves, and it enables to retrieve expected rates of convergence.

We expect the model to be able to handle such flows as those encountered in the boiling crisis and in some other specific situations occuring in the framework of nuclear safety analysis. We also refer to the paper $^{19}$, that presents some preliminary results of the flow along a heated wall, which have been obtained in a $2 \mathrm{D}$ framework with the $\mathrm{BN}$ model.

\section{Acknowledgments:}

The third author receives some financial support by ANRT under grant CIFRE/EDF 2009/529.

\section{References}

1 Ambroso, A., Chalons, C., Coquel, F., and Galié, T., "Relaxation and numerical approximation of a two-fluid two-pressure diphasic model" Math. Model. and Numer. Anal., vol. 43(6), 2009, pp. 1063-1098.

2 Ambroso, A., Chalons, C., And Raviart, P.A., "A Godunov-type method for the seven-equation model of compressible two-phase flow" Computers and Fluids, vol.54, 2012, pp. 67-91.

3 Andrianov, N., And Warnecke, G., " The Riemann problem for the Baer-Nunziato two-phase flow model", J. Comp. Physics., vol. 195, 2004, pp. 434-464.

4 Baer, M.R., And Nunziato, J.W., "A two-phase mixture theory for the deflagration to detonation transition (DDT) in reactive granular materials", Int. J. Multiphase Flow, vol. 12(6), 1986, pp. 861-889.

5 Bdzil, J.B., Menikoff, R., Son, S.F., Kapila, A.K., and Stewart, D.S., "Two-phase modeling of a DDT in granular materials: a critical examination of modeling issues", Phys. of Fluids, vol. 11, 1999, pp. 378-402.

6 Coquel, F., Galloü̈t, T., Hérard, J.M., and Seguin, N., "Closure laws for a two-fluid two-pressure model", $C$. $R$. Acad. Sci. Paris, vol. I-332, 2002, pp. 927-932.

7 Crouzet, F., Daude, F., Galon, P., Helluy, P., Hérard, J.-M., and Liu, Y., "On the computation of the BaerNunziato model", contribution to the 42th AIAA FD conference, 2012.

8 Drew, D.A, And Passman, M., "Theory of multicomponent fluids", Applied Mathematical Sciences, vol. 135, Springer, 1999.

9 Embid, P., And BAER, M., "Mathematical analysis of a two-phase continuum mixture theory", Contin. Mech. Thermodyn., vol. 4, 1992, pp. 279-312.

10 Gallouët, T., Helluy, P., Hérard, J.-M., and Nussbaum J., "Hyperbolic relaxation models for granular flows", Math. Model. and Numer. Anal., vol.44(2), 2010, pp.371-400.

11 Gallouët, T., Hérard, J.-M., and Seguin, N., "Numerical modelling of two phase flows using the two-fluid twopressure approach", Math. Mod. Meth. in Appl. Sci., vol. 14(5), 2004, pp. 663-700. 
12 Gavrilyuk, S., And Saurel, R., "Mathematical and numerical modelling of two phase compressible flows with inertia", J. Comp. Physics., vol. 175, 2002, pp. 326-360.

13 Girault, L., And HÉrard, J.-M., "A two-fluid hyperbolic model in a porous medium", Math. Model. and Numer. Anal., vol. 44(6), 2010, pp. 1319-1348.

14 Glimm, J., Saltz, D., AND Sharp, D.H., "Renomalization group solution of two-phase flow equations for Rayleigh Taylor mixing", Phys. Lett., vol. A222, 1996, pp. 171-1763.

15 Glimm, J., Saltz, D., And Sharp, D.H., "Two phase flow modelling of a fluid mixing layer", J. Fluid Mechanics, vol. 378, 1999, pp. 119-143.

16 Gonthier, K.A., And Powers, J.M., "A high resolution method for a two-phase flow model of deflagration to detonation transition ", J. Comp. Physics., vol. 163, 2000, pp.376-433.

17 HÉrard, J.-M., "A three-phase flow model", Mathematical Computer Modelling, vol. 45, 2007, pp. 432-455.

18 HÉRARD, J.-M., "Une classe de modèles diphasiques bi-fluides avec changement de régime", internal EDF report H-I812010-0486-FR, in French, 2010.

19 HÉrard, J.-M., AND Hurisse, O., "Computing two-fluid models of compressible water-vapour flows with mass transfer", contribution to the 42th AIAA FD conference, 2012.

20 IshiI, M., "Thermofluid dynamic theory of two-phase flow", Collection de la Direction des Etudes et Recherches d'Electricité de France, Collection Eyrolles, 1975.

21 Jin, H., And Glimm, J., "Weakly compressible two-pressure two-phase flow", Acta Mathematica Scienta, vol. 29, 2009, pp. $1497-1540$

22 Kapila, A.K., Menikoff, R., Bdzil, J.B., Son, S.F., And Stewart, D.S., "Two-phase modeling of a DDT in granular materials: reduced equations", Phys. of Fluids, vol. 13, 2001, pp. 3002-3024.

23 Kapila, A.K., Son, S.F., Bdzil, J.B., Menikoff, R., And Stewart, D.S., "Two-phase modeling of a DDT: structure of the velocity relaxation zone", Phys. of Fluids, vol. 9(12), 1997, pp. 3885-3897.

24 Lhuillier, D., "A mean field description of two-phase flows with phase changes", Int. J. of Multiphase Flow, vol. 29, 2003, pp. 511-525.

25 Lhuillier, D., "Evolution of the volumetric interfacial area in two-phase mixtures", C. R. Mécanique, vol. 332, 2004, pp. 103-108.

26 LiU, Y., PhD thesis, Université Aix-Marseille I, Marseille, France, in preparation, 2013.

27 Lowe, C.A., " Two-phase shock-tube problems and numerical methods of solution", J. Comp. Physics., vol. 204, 2005, pp. 598-632.

28 Papin, M., And Abgrall, R., "Fermetures entropiques pour les systèmes bifluides à sept équations", C. R. Mécanique, vol. 333,2005 , pp. $838-842$.

29 Saleh, K., PhD thesis, Université Pierre et Marie Curie, Paris, France, in preparation, 2012.

30 Saurel, R., And Abgrall, R., " A Multiphase Godunov Method for Compressible Multifluid and Multiphase Flows ", J. Comp. Physics., vol. 150, 1999, pp. 425-467.

31 Schwendeman, D.W., Wahle, C.W., and Kapila, A.K., " The Riemann problem and a high-resolution Godunov method for a model of compressible two-phase flow ", J. Comp. Physics., vol. 212, 2006, pp. 490-526.

32 Schwendeman, D.W., Kapila, A.K., and Henshaw, W.D., " A study of detonation diffraction and failure for a model of compressible two-phase reactive flow ", Combustion Theory and Modelling, vol. 14, 2010, pp. 331-366.

33 Tokareva, S.A., ANd Toro, E.F., " HLLC type Riemann solver for the Baer-Nunziato equations of compressible two-phase flow", J. Comp. Physics., vol. 229, 2010, pp. 3573-3604. 